Pacific Journal of Mathematics

ON SOME MAPPINGS RELATED TO GRAPHS 


\section{ON SOME MAPPINGS RELATED TO GRAPHS}

\section{PaUl Kelly}

Let $N$ denote a set of $n$ distinct elements $a_{1}, a_{2}, \ldots, a_{n}$ and let $\mathscr{S}(h)=\left\{S_{1}, S_{2}, \cdots, S_{m}\right\}, m=\left(\begin{array}{l}n \\ h\end{array}\right)$ be the collection of all sets formed by selecting $h$ elements at a time from $N$. If $S_{i}=\left\{a_{i_{1}}, a_{i_{2}}, \cdots, a_{i_{h}}\right\}$ is any set in $\mathscr{S}(h)$ and if $\Gamma$ is any mapping of $N$ onto itself, then $\Gamma$ induces a mapping $\Psi$ of $\mathscr{S}(h)$ onto itself defined by $S_{i} \Psi=$ $\left\{a_{i_{1}} \Gamma, a_{i_{2}} \Gamma, \cdots, a_{i_{h}} \Gamma\right\}$. We seek conditions under which, conversely, a mapping of $\mathscr{S}(h)$ onto itself must be of this induced type.

If $\Psi$ is a mapping of $\mathscr{S}(h)$ onto itself, it will be said to "preserve maximal intersections" if each two of its sets which intersect on $h-1$ elements are mapped to two sets which also have $h-1$ elements in common. It will be shown that if $n \neq 2 h$ this is sufficient to imply that $\Psi$ is induced by a mapping of $N$ onto itself.

We observe first that to each set $S_{i}$ in $\mathscr{S}(h)$ there corresponds a set $S_{1}^{*}$ in $\mathscr{S}(n-h)$ and which consists of those elements of $N$ not in $S_{i}$. And to any mapping $\Psi$ of $\mathscr{S}(h)$ onto itself there corresponds a mapping $\Psi^{*}$ of $\mathscr{S}(n-h)$ onto itself defined by $S_{i}^{*} \Psi^{*}=\left(S_{i} \Psi\right)^{*}, i=$ $1,2, \cdots, m$. Clearly, if $\Psi$ preserves maximal intersections so does $\Psi^{*}$ and both $\Psi$ and $\Psi^{*}$ are induced mappings or neither is. Thus it suffices always to consider the case $h \leqq n-h$, that is, $h \leqq n / 2$.

THEOREM 1. If $n \neq 2 h$ and if $\Psi$ is a mapping of $\mathscr{S}(h)$ onto itself which preserves maximal intersections, then $\Psi$ is induced by a mapping of $N$ onto itself.

Proof. The theorem is trivially correct for $h=1$. For a proof by induction, we suppose the theorem true up to some value $h-1$ and consider $\Psi$ to be a mapping of $\mathscr{S}(h)$ onto itself, where $1<h<n / 2$.

Each set in $\mathscr{S}(h-1)$ belongs to exactly $n-h+1$ sets in $\mathscr{S}(h)$ and we wish to show that these sets in $\mathscr{S}(h)$ must map under $\Psi$ to $n-h+1$ sets which also have a set of $h-1$ elements in common. Suppose that this is not the case. Then there exists a set in $\mathscr{S}(h-1)$, which we may take to be $T=\left\{a_{1}, a_{2}, \cdots, a_{h-1}\right\}$, such that the sets in $\mathscr{S}(h)$ which contain $T$ do not map under $\Psi$ to a collection of sets with a common intersection of $h-1$ elements. Let

$$
S_{i}=\left\{a_{1}, a_{2}, \cdots, a_{h-1}, a_{h+i}\right\}, \quad i=0,1, \cdots, h, \cdots, n-h
$$

denote the sets of $\mathscr{S}(h)$ which contain $T$. There is no loss of gener-

Received March 20, 1963. This work was supported by Contract NSF-G23718. 
ality in supposing that it is the intersection of $S_{0} \Psi$ and $S_{1} \Psi$ which is not contained in $S_{2} \Psi$. Since $\Psi$ preserves maximal intersections, we can denote

$$
S_{0} \Psi=\left\{b_{1}, b_{2}, \cdots, b_{h-1}, b_{h}\right\}, \quad S_{1} \Psi=\left\{b_{1}, b_{2}, \cdots, b_{h-1}, b_{h+1}\right\},
$$

where each $b_{i}$ is an element from $N$ and $i \neq j$ implies $b_{i} \neq b_{j}, i, j=$ $1,2, \cdots, h+1$. Because $S_{2} \Psi$ does not contain $\left\{b_{1}, b_{2}, \cdots, b_{h-1}\right\}$, but must intersect $S_{0} \Psi$ and $S_{1} \Psi$ on $h-1$ elements, $S_{2} \Psi$ must contain both $b_{h}$ and $b_{h+1}$ and fail to possess just one elements from $b_{1}, b_{2}, \cdots, b_{h-1}$. Since there is nothing to distinguish the possibilities, we may suppose that $S_{2} \Psi$ does not possess $b_{1}$, and hence that

$$
S_{2} \Psi=\left\{b_{2}, \cdots, b_{h-1}, b_{h}, b_{h+1}\right\}
$$

Because $n>2 h$, there are at least $h+2$ sets $S_{i}$ defined by (1) and so at least $h-1$ sets $S_{i}$, where $2<i \leqq n-h$. And the $\Psi$ images of all these sets must possess $b_{1}, b_{h}$, and $b_{h+1}$. For suppose $b_{1} \notin S_{i} \Psi$. Since $S_{i} \Psi$ intersects $S_{0} \Psi$ on $h-1$ elements and not on $b_{1}$ then $\left\{b_{2}, b_{3}, \cdots, b_{h}\right\} \subset S_{i} \Psi$. And since $S_{i} \Psi$ intersects $S_{1} \Psi$ on $h-1$ elements and not on $b_{1}$, then $\left\{b_{2}, \cdots, b_{h}, b_{h+1}\right\} \subset S_{i} \Psi$. But then $S_{i} \Psi=$ $\left\{b_{2}, \cdots, b_{h}, b_{h+1}\right\}=S_{2} \Psi$, which is impossible for $i \neq 2$. In the same way, $b_{h} \notin S_{i} \Psi$ implies $S_{i} \Psi=S_{1} \Psi$ and $b_{h+1} \notin S_{i} \Psi$ implies $S_{i} \Psi=S_{0} \Psi$, neither of which is possible for $2<i \leqq n-h$.

From the last argument it follows that for $i>2, S_{i} \Psi$ must be of the form

$$
S_{i} \Psi=\left\{b_{1}, b_{h}, b_{h+1}, x_{1}, \cdots, x_{h-3}\right\},
$$

where $\left\{x_{1}, x_{2}, \cdots, x_{h-3}\right\}$ is a subset of $\left\{b_{2}, b_{3} \cdots, b_{h-1}\right\}$, which is clearly impossible if $h=2$. But in any case, there are at least $h-1$ different sets $S_{i} \Psi$, where $i>2$, and each of these is determined by the $h-3$ order subset of $\left\{b_{2}, \cdots, b_{h-1}\right\}$ which it contains. And since there are only $h-2$ mutually different such subsets, the sets $S_{i} \Psi, i>2$, cannot all be distinct, which contradicts the fact that $\Psi$ is a one-to-one mapping.

It is now established that for each set $T$ in $\mathscr{S}(h-1)$ there exists a set $T^{\prime}$ in $\mathscr{S}(h-1)$ such that all the sets in $\mathscr{S}(h)$ which contain $T$ are mapped under $\Psi$ to all the sets in $\mathscr{S}(h)$ which contain $T^{\prime}$. But then the correspondence $T \rightarrow T^{\prime}$ is clearly a mapping of $\mathscr{S}(h-1)$ onto itself, say the mapping $\Phi$.

For $h=2, \Phi$ is a mapping of $N$ onto itself. If $\left\{a_{i}, a_{j}\right\}$ is any set in $\mathscr{S}(2)$, then $a_{i} \Phi$ belongs to the $\Psi$ images of all sets which possess $a_{i}$, so $a_{i} \Phi$ belongs to $\left\{a_{i}, a_{j}\right\} \Psi$. By the same argument, $a_{j} \Phi$ 
belongs to $\left\{a_{i}, a_{j}\right\} \Psi$. Since $a_{i} \Phi \neq a_{j} \Phi$, it follows that $\left\{a_{i}, a_{j}\right\} \Psi=\left\{a_{i} \Phi, a_{j} \Phi\right\}$ and hence that $\Psi$ is induced by $\Phi$.

If $h>2$, consider any two sets in $\mathscr{S}(h-1)$, whose intersection is maximal, say

$$
T_{1}=\left\{\alpha_{1}, a_{2}, \cdots, a_{h-2}, a_{h-1}\right\}, \quad T_{2}=\left\{\alpha_{1}, a_{2}, \cdots, a_{h-2}, a_{h}\right\} .
$$

The set $S=\left\{a_{1}, a_{2}, \cdots, a_{h}\right\}$ in $\mathscr{S}(h)$ maps to a set $S \Psi=\left\{b_{1}, b_{2}, \cdots, b_{h}\right\}$. Since $T_{1}$ and $T_{2}$ are contained in $S, T_{1} \Phi$ and $T_{2} \Phi$ are $h-1$ order subsets of $S \Psi$. Since $T_{1} \neq T_{2}$, and $\Phi$ is a one-to-one mapping, $T_{1} \Phi \neq T_{2} \Phi$, so the order of $T_{1} \Phi \cap T_{2} \Phi$ is $h-2$. Thus $\Phi$ preserves maximal intersections and so, by the inductive hypothesis, $\Phi$ is induced by some mapping $\Gamma$ of $N$ onto itself.

Now $S=\left\{a_{1}, a_{2}, \cdots, a_{h}\right\}$ contains $T_{1}$ and $T_{2}$ defined in (5) so $S \Psi$ contains $T_{1} \Phi$ and $T_{2} \Phi$. But $T_{1} \Phi=\left\{a_{1} \Gamma, a_{2} \Gamma, \cdots, a_{h-1} \Gamma\right\}$, and $T_{2} \Phi=$ $\left\{a_{1} \Gamma, \cdots, a_{h-1} \Gamma, a_{h} \Gamma\right\}$. Since $a_{i} \Gamma \neq a_{j} \Gamma$ if $i \neq j$, it follows that $S \Psi=$ $\left\{a_{1} \Gamma, a_{2} \Gamma, \cdots, a_{h} \Gamma\right\}$, and hence that $\Psi$ is induced by $\Gamma$.

The theorem is not true for $n=2 h$, since then the correspondence of $S_{i}$ and $S_{i}^{*}$ is a non-induced mapping of $\mathscr{S}(h)$ onto itself which preserves all orders of intersection. ${ }^{1}$

Consider next an ordinary, finite graph $G$, that is, one with $n$ vertices $\left\{p_{1}, p_{2}, \cdots, p_{n}\right\}$ where each two vertices have at most one join and none is joined to itself. Let $c\left(p_{i}, p_{j}, p_{k}\right)$ denote the subgraph of $G$ induced by $G$ on the set of vertices which does not include $p_{i}, p_{j}, p_{k}$, and let $m(G)$ be the notation for the join-measure of $G$, that is the number of joins in $G$.

THeOREM 2. If $G$ and $H$ are ordinary nth order graphs and if there is a mapping of the vertices of $G$ onto those of $H$ such that for some integer $h, 1<h<n-1$, all corresponding subgraphs of order $h$ have the same join measure, then the mapping is an isomorphism of $G$ and $H$.

Proof. For $h=2$ the condition becomes the definition of an isomorphism, so assume that $2<h<n-1$. Let $\left\{p_{1}, p_{2}, \cdots, p_{n}\right\}$ be the vertices of $G$ and let the vertices $\left\{q_{1}, q_{2}, \cdots, q_{n}\right\}$ of $H$ be labeled so that $q_{i}$ is the image of $p_{i}$ under the given mapping $\psi, i=1,2, \cdots, n$.

Let $\left\{p_{i_{1}}, p_{i_{2}}, \cdots, p_{i_{h+1}}\right\}$ be the vertices of any subgraph $G_{i}$ of order $h+1$ in $G$, and let $c\left(p_{i_{k}} ; G_{i}\right)$ denote the subgraph of $G_{i}$ defined on all the vertices of $G_{i}$ except $p_{i_{k}}$. Since any join in $G_{i}$ belongs to all the $h$-order subgraphs of $G_{i}$ except two, we have,

$$
m\left(G_{i}\right)=\frac{1}{h-1} \sum_{k=1}^{k=h+1} m\left[c\left(p_{i_{k}} ; G_{i}\right)\right] .
$$

This general exception was pointed out to the writer by P. Erdös. 
By the same reasoning,

$$
m\left(G_{i} \Psi\right)=\frac{1}{h-1} \sum_{k=1}^{k=h+1} m\left[c\left(q_{i_{k}} ; G_{i} \Psi\right)\right]
$$

Since, by assumption,

$$
m\left[c\left(p_{i_{k}} ; G_{i}\right)\right]=m\left[c\left(q_{i_{k}} ; G_{i} \Psi\right)\right], \quad \text { for all } p_{i_{k}} \text { and } q_{i_{k}},
$$

it follows that $m\left(G_{i}\right)=m\left(G_{i} \Psi\right)$.

Thus if $\Psi$ preserves the join measure on $h$-order subgraphs it does so on $h+1$ order subgraphs, and, by the same reasoning, preserves the join measure on all subgraphs of order equal to or greater than $h$. In particular, $m(G)=m(H)$. Then if $\rho\left(p_{i}\right)$ denotes the degree of $p_{i}$, it follows from

$$
\rho\left(p_{i}\right)=m(G)-m\left[c\left(p_{i}\right)\right], \quad i=1,2, \cdots, n
$$

and

$$
\rho\left(q_{i}\right)=m(H)-m\left[c\left(q_{i}\right)\right], \quad i=1,2, \cdots, n
$$

that

$$
\rho\left(p_{i}\right)=\rho\left(q_{i}\right),
$$$$
i=1,2, \cdots, n \text {, }
$$

since $m\left[c\left(p_{i}\right)\right]=m\left[c\left(q_{i}\right)\right]$.

Now, corresponding to $p_{i}$ and $p_{j}$ in $G$, let $\varepsilon_{i j}$ be 1 or 0 according as $p_{i}$ and $p_{j}$ are or are not joined. Let $\varepsilon_{i j}^{\prime}$ be defined in a similar way with respect to $q_{i}$ and $q_{j}$. Then, by simple counting,

$$
m(G)=m\left[c\left(p_{i}, p_{j}\right)\right]+\rho\left(p_{i}\right)+\rho\left(p_{j}\right)-\varepsilon_{i j}, \quad i \neq j,
$$

and

$$
m(H)=m\left[c\left(q_{i}, q_{j}\right)\right]+\rho\left(q_{i}\right)+\rho\left(q_{j}\right)-\varepsilon_{i j}^{\prime}, \quad i \neq j .
$$

Comparing the terms in (7) and (8) it follows that $\varepsilon_{i j}=\varepsilon_{i j}^{\prime}$ for all $i, j$, $i \neq j$, and hence that $\Psi$ is an isomorphism of $G$ and $H$.

As a corollary of these theorems it follows that two $n$th order graphs are isomorphic if and only if there is a one-to-one correspondence of their subgraphs of some order $h, 1<h<n-1$, in which corresponding subgraphs have equal join measure and the correspondence preserves maximal intersections.

University of CALIForNia, SANTA Barbara 


\section{PACIFIC JOURNAL OF MATHEMATICS}

\section{EDITORS}

Robert Osserman

Stanford University

Stanford, California

M. G. Arsove

University of Washington

Seattle 5 , Washington
J. DugundjI

University of Southern Califorma: Los Angeles 7, California

Lowell J. Paige

University of California

Los Angeles 24, California

\section{ASSOCIATE EDITORS}

E. F. BECKENBACH

B. H. NeumanN

F. WOLF

K. YosIDA

\section{SUPPORTING INSTITUTIONS}

UNIVERSITY OF BRITISH COLUMBIA

CALIFORNIA INSTITUTE OF TECHNOLOGY

UNIVERSITY OF CALIFORNIA

MONTANA STATE UNIVERSITY

UNIVERSITY OF NEVADA

NEW MEXICO STATE UNIVERSITY

OREGON STATE UNIVERSITY

UNIVERSITY OF OREGON

OSAKA UNIVERSITY

UNIVERSITY OF SOUTHERN CALIFORNIA
STANFORD UNIVERSITY

UNIVERSITY OF TOKYO

UNIVERSITY OF UTAH

WASHINGTON STATE UNIVERSITY

UNIVERSITY OF WASHINGTON

AMERICAN MATHEMATICAL SOCIETY CALIFORNIA RESEARCH CORPORATION SPACE TECHNOLOGY LABORATORIES NAVAL ORDNANCE TEST STATION 


\section{Pacific Journal of Mathematics}

\section{Vol. 14, No. 1 \\ May, 1964}

Richard Arens, Normal form for a Pfaffian .........................

Charles Vernon Coffman, Non-linear differential equations on cones in Banach

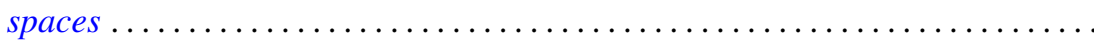

Ralph DeMarr, Order convergence in linear topological spaces ..............

Peter Larkin Duren, On the spectrum of a Toeplitz operator ................

Robert E. Edwards, Endomorphisms of function-spaces which leave stable all

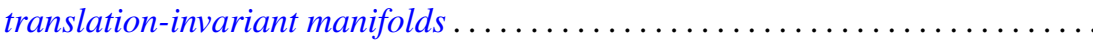

Erik Maurice Ellentuck, Infinite products of isols . . . . . . . . . . . . . . . . 49

William James Firey, Some applications of means of convex bodies . . . . . . . . 53

Haim Gaifman, Concerning measures on Boolean algebras ............. 61

Richard Carl Gilbert, Extremal spectral functions of a symmetric operator. . . . . . 75

Ronald Lewis Graham, On finite sums of reciprocals of distinct nth powers ..... 85

Hwa Suk Hahn, On the relative growth of differences of partition functions ...... 93

Isidore Isaac Hirschman, Jr., Extreme eigen values of Toeplitz forms associated

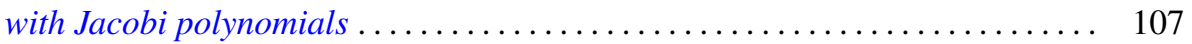

Chen-jung Hsu, Remarks on certain almost product spaces . . . . . . . . . . . 163

George Seth Innis, Jr., Some reproducing kernels for the unit disk . . . . . . . . . 177

Ronald Jacobowitz, Multiplicativity of the local Hilbert symbol . . . . . . . . . . . 187

Paul Joseph Kelly, On some mappings related to graphs ................. 191

William A. Kirk, On curvature of a metric space at a point . . . . . . . . . . . . 195

G. J. Kurowski, On the convergence of semi-discrete analytic functions . . . . . . . 199

Richard George Laatsch, Extensions of subadditive functions . . . . . . . . . . . 209

V. Marić, On some properties of solutions of $\Delta \psi+A\left(r^{2}\right) X \nabla \psi+C\left(r^{2}\right) \psi=0 \ldots 217$

William H. Mills, Polynomials with minimal value sets . . . . . . . . . . . 225

George James Minty, Jr., On the monotonicity of the gradient of a convex

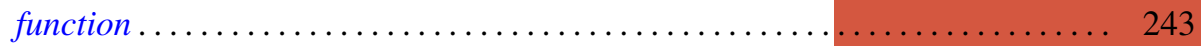

George James Minty, Jr., On the solvability of nonlinear functional equations of 'monotonic' type ................................... 249

J. B. Muskat, On the solvability of $x^{e} \equiv e(\bmod p) \ldots \ldots \ldots \ldots \ldots \ldots \ldots \ldots . \ldots \ldots$

Zeev Nehari, On an inequality of $P . R$. Bessack ................... 261

Raymond Moos Redheffer and Ernst Gabor Straus, Degenerate elliptic

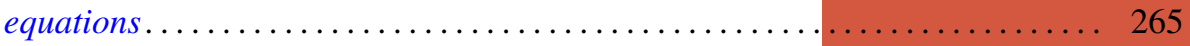

Abraham Robinson, On generalized limits and linear functionals . . . . . . . . . 269

Bernard W. Roos, On a class of singular second order differential equations with a

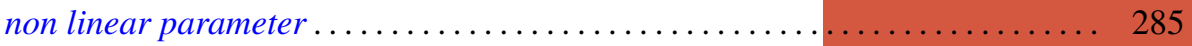

Tôru Saitô, Ordered completely regular semigroups . . . . . . . . . . . . . . . . 295

Edward Silverman, A problem of least area ....................... 309

Robert C. Sine, Spectral decomposition of a class of operators . . . . . . . . . 333

Jonathan Dean Swift, Chains and graphs of Ostrom planes . . . . . . . . . . . 353

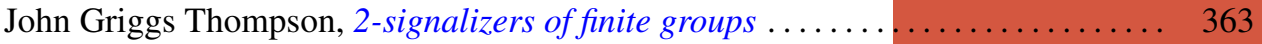

Harold Widom, On the spectrum of a Toeplitz operator . . . . . . . . . . . . . 365 